\title{
A Study of r- and u-Magnitude Dependence in the Spatial Orientation of Spin Vectors of SDSS Galaxies having redshift $0.10<\mathrm{z}<0.11$
}

\author{
Shiv Narayan Yadav \& Binil Aryal \\ Central Department of Physics, Tribhuvan University, Kirtipur
}

\begin{abstract}
We present a study of spin vector orientations of $44749 r$ and u-magnitude SDSS (Sloan Digital Sky Survey) galaxies having redshift 0.10 to 0.11. The $r$ and u-magnitudes are observed through $616.5 \mathrm{~nm}$ and $354.3 \mathrm{~nm} C C D$ (charge coupled device) filter attached to SDSS telescope located at New Mexico, USA. We have converted two dimensional data to three dimensional galaxy rotation axes. Our aim is to find out non-random effects in the spatial orientation of galaxies. In addition, we wanted to check $r$ and u-magnitude dependence in the spatial orientation. The expected isotropy distribution curves are obtained by removing the selection effects and performing a random simulation method. In general, spin vector orientations of galaxies is found to be random, supporting Hierarchy model of galaxy formation. A local anisotropy is observed in few samples suggesting a gravitational tidal interaction between neighbor galaxies, a merging process in which the angular momentum vector distort the original alignment of evolution of rotational supported system to the randomized system.
\end{abstract}

Keywords: clusters: Supercluster -- galaxies: formation, evolution -- galaxies: statistics -- galaxies: clusters: general -astronomical databases: miscellaneous

\section{INTRODUCTION}

Modern cosmology is based on two fundamental assumptions: First, the dominant interaction on cosmological scales is gravity, and second, the cosmological principle is a good approximation to the universe. The cosmological principle states that the universe, smoothed over large enough scales, is essentially homogeneous and isotropic. 'Homogeneity' has the intuitive meaning that at a given time the universe looks the same everywhere, and 'isotropy' refers to the fact that for any observer moving with the local matter the universe looks (locally) the same in all directions. On the other hand, huge low redshift galaxy surveys such as the 2-degree field galaxy redshift survey (2dFGRS, Colless et al. 2001) and the Sloan Digital Sky Survey (SDSS, York et al. 2000) have convinced most cosmologists that not only isotropy but also homogeneity is in fact a reasonable assumption for the universe. The Universe is homogeneous and isotropic on scales larger than $100 \mathrm{Mpc}$, but on smaller scales we observe huge deviations from the mean density in the form of galaxies, galaxy clusters, and the cosmic web being made of sheets and filaments of galaxies. How do structures grow in the universe and how can we describe them? The most accepted view on the formation and evolution of large scale structure is that it was formed as a consequence of the growth of primordial fluctuations by gravitational instability. In the current favored model, smaller structures collapse first and are later incorporated in larger collapsing structures in a bottom-up scenario that provides a natural explanation for the formation of galaxies, clusters, filaments and superclusters.

Galaxy clusters are gravitationally bound large scale structures in the universe. To understand the evolution of these aggregates it is essential to know when and how they have formed and how their structures and constituents have been changing with time. Gamow (1952) made it clear that the observed rotations of galaxies are important for cosmology. According to them, the fact that galaxies rotate might be a clue of physical conditions under which these systems formed. Thus, understanding the distribution of spatial orientations of the spin vectors (hereafter SVs) or angular momentum vectors of galaxies is very important. It could allow us to know the origin of angular momenta of galaxies.

There are three predictions about the spatial 
orientation of spin vectors of galaxies. These are the 'pancake model', the 'hierarchy model,' and the 'primordial vorticity theory.' The 'pancake model' (Doroshkevich 1973; Doroshkevich \& Shandarin 1978) predicts that the spin vectors (SVs hereafter) of galaxies tend to lie within the cluster plane. According to the 'hierarchy model' (Peebles 1969), the directions of the SVs should be distributed randomly. The 'primordial vorticity theory' (Ozernoy 1971, 1978; Stein 1974) predicts that the spin vectors of galaxies are distributed primarily perpendicular to the cluster plane. In the present work we intend to work on SDSS ( $7^{\text {th }}$ data release) database of galaxies that have radial velocity in the range $30,000 \mathrm{~km} / \mathrm{s}$ to $33,000 \mathrm{~km} / \mathrm{s}(0.10<z<0.11)$. We are interested to check whether magnitude dependence exist concerning galaxy orientation or not.

\section{SDSS PHOTOMETRY}

The distribution of $r$ and $u$ magnitudes gives important information regarding the process of star formation activity in the star. The apparent magnitudes do not tell us about the true brightness of stars, since the distances differ. A quantity measuring the intrinsic brightness of a star is the absolute magnitude. The absolute magnitude $M$ of any star is defined as the apparent magnitude at a distance

of $10 \mathrm{pc}$ from the star in the absence of astronomical extinction.

We know that the relation between magnitude, distance and opacity is given by (Padmanabhan 2006),

$$
m-M=5 \log r-5+(2.5 \log e) a r
$$

Here $m, M$ and $\alpha$ represent apparent, absolute magnitudes and opacity of the medium.

For $r$ and $u$ filter,

$$
\begin{aligned}
& m_{r}-M_{r}=5 \log r-5+(2.5 \log e) a_{r} r \\
& m_{u}-M_{u}=5 \log r-5+(2.5 \log e) a_{u} r
\end{aligned}
$$

Subtracting equation (3) from (2) we get, $r=\left[\left(m_{r}-m_{u}\right)-\left(M_{r}-M_{u}\right)\right] /\left[2.5 \log e\left(a_{r}-a_{u}\right)\right]$
This equation is useful to calculate the value of distance to the galaxy. Thus, the magnitude through various filters gives precise information about distance.


Figure 1: Distribution of galaxies through two $(r$ $\& u)$ filters. The observed distribution with $\pm 1 \sigma$ error bars can be seen. The solid lines represent the Gaussian fit.

In our database we have included the apparent magnitude of the galaxies. Magnitude distribution for $u$ and $r$ filters is shown as in Fig. 1. We found that the observed magnitude distribution of galaxies for the $u$-filter fits well with the Gaussian distribution but for $r$ filters, growing function is normal but decaying function is sharply falling (see Fig. 1).

\section{DATA COMPILATION}

We use the database of SDSS galaxies that have redshift in the range 0.10 to 0.11 . These database were downloaded from Sloan Digital Sky Survey (SDSS) Data Release 7. There are 44,830 galaxies in the region of interest.

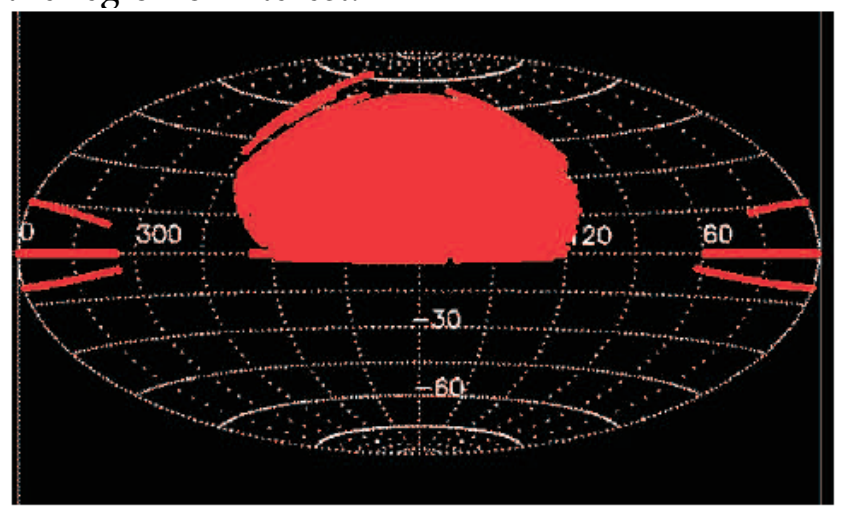






Figure 2: All sky distribution of galaxies covered by SDSS $7^{\text {th }}$ data release in the equatorial co-ordinate system (left). Wavelength bands of the filters used by SDSS telescope (right). The transmittance through $\mathrm{u}$-filter is relatively weak compared to that of other filters because of the absorption feature of Earth's atmosphere.

Figure 2 shows the all sky distribution of galaxies of our database. The apparent distribution of the galaxies is because of the nature of the survey. Acharya (2012) carried out a study on the same database and found that the optical search limit is complete for the galaxies that have major diameter $a>24$ arc minutes. We plotted bin size versus standard error of the database and use basic statistic and obtained the appropriate bin size in order to classify subsamples.

\section{GODLOWSKIAN TRANSFORMATION}

The three dimensional orientation of the SV of a galaxy is characterized by two angles: the polar angle $(\theta)$ between the galactic SV and a reference plane (here equatorial plane), and the azimuthal angle $(\phi)$ between the projection of a galactic SV on to this reference plane and the $\mathrm{X}$-axis within this plane. The detail derivations of the expressions of the angles $\theta$ and $\phi$ are given in Flin \& Godłowski (1986). When using equatorial coordinate system as reference, then $\theta$ and $\phi$ can be obtained from measurable quantities as follows:

$\ddot{\sin } \theta=-\dot{\cos } i \sin L \pm \dot{\sin } i \dot{\sin } P \dot{\mathrm{c} o \mathrm{~s}} B$ $\sin \phi=(\dot{\cos } \theta)^{-1}[-\dot{c o s} i \cos B \sin L+\sin i(\mp \sin P \sin B \sin L \mp \dot{c o s} P \cos L)]$

where $i$ is the inclination angle, the angle between the normal to the galaxy plane and the observer's line-of-sight. The inclination angle can be computed from the formula

$$
\dot{\cos } \dot{\mathrm{s}}^{2} i=\frac{\left(q^{2}-q^{*^{2}}\right)}{\left(1-q^{*^{2}}\right)}
$$

This expression is valid for oblate spheroids (Holmberg 1946). Here, $q$ and $q^{*}$ represent the measured axial ratio $(b / a)$ and the intrinsic flatness of the galaxy, respectively. The intrinsic flatness of a disk galaxy depends on the morphological type and is taken from Heidmann et al. (1971). Hiedmann et al. (1971) showed that the values of $q^{*}$ range from 0.083 for Sd spirals to 0.33 for ellipticals. For the galaxies with unknown morphology $q^{\star}=0.20$ is assumed.

\section{NUMERICAL SIMULATION}

Here we describe the procedure for the removal the selection effects to obtain the isotropic distributions for both $\theta$ and $\phi$ as given by Aryal \& Saurer (2000). Theoretically, the isotropic distribution curve for polar angle is cosine and that for azimuthal angle is the average distribution curve, with the restriction that the database is free from se lection effect. Aryal \& Saurer (2000) concluded that any selections imposed on the database may cause severe changes in the shapes of the expected isotropic distribution curves. In their method, a true spatial distribution of the galaxy rotation axis is assumed to be isotropic. Then, due to the projection effects, $i$ can be distributed as $\sin i, B$ can be distributed $\cos B$, the variables $L$ and $P$ can be distributed randomly, and the equation $(5,6)$ can be used to calculate the corresponding values of polar $(\theta)$ and azimuthal $(\phi)$. We run simulations in order to define expected isotropic distribution curves for both the $\theta$ and $\phi$ distributions. The isotropic distribution curves are based on simulations including $10^{7}$ virtual galaxies. At first we observed the distributions of L, B, P and 
i for the galaxies in our samples and distributed by creating $10^{7}$ virtual galaxies for respective parameters. We use these numbers to make input file and the expected distribution by running simulation in MATLAB 7.0. The program file is as follows:

name of the program__e: polarl

clear all; \% making the work place free of memory

$\mathrm{t}=$ cputime;

input(Type the name of your input_le: ):

deltal $=\operatorname{asin}\left(\_\cos (\mathrm{i}){ }^{*}{ }^{*} \sin (\mathrm{B})+\sin (\mathrm{i}){ }^{*} \cdot \sin (\mathrm{P}){ }^{*} \cos (\mathrm{B})\right) ; \%$ polar angle delta2 = $\operatorname{asin}\left(\_\cos (\mathrm{i}){ }^{*}{ }^{*} \sin (\mathrm{B}) \_\sin (\mathrm{i}){ }^{*} \sin (\mathrm{P}){ }^{*}{ }^{*} \cos (\mathrm{B})\right) ; \%$ \%plar angle

etal $=\operatorname{asin}\left(\left(\cos (\mathrm{i}){ }^{*} \cos (\mathrm{B}) \cdot{ }^{*} \sin (\mathrm{L})+\sin (\mathrm{i}) .{ }^{*}(\right.\right.$ $\left.\left.\sin (P) .{ }^{*} \sin (B) .{ }^{*} \sin (L) \_\cos (P) .{ }^{*} \cos (L)\right)\right) . / \cos ($ thetal)); \%azimuthal angle

eta2 $=\operatorname{asin}\left(\left(\cos (\mathrm{i}){ }^{*} \cos (\mathrm{B}){ }^{*} \cdot{ }^{*} \sin (\mathrm{L})+\sin (\mathrm{i}){ }^{*}{ }^{*}+\sin (\mathrm{P}){ }^{*} \sin (\mathrm{B}){ }^{*} \mathrm{~s}\right.\right.$ in $(\mathrm{L})+$

$\left.\left.\cos (\mathrm{P}) .{ }^{*} \cos (\mathrm{L})\right)\right) . / \cos ($ thetaZ)); \%azimuthal angle

deltal $=$ deltal. ${ }^{*} \mid ⿰ \mathrm{C} \mathrm{D} / \mathrm{pi} ; \%$ changing to degrees

deltaZ = deltaZ. ${ }^{*} \mid 80 / p$ i;

etal $=$ etal. ${ }^{*} \mid 80 / \mathrm{pi} ;$

etaZ = eta2. ${ }^{*} 180 / p$;

delta=[deltal; delta2]; \%polar angle

eta=[etal; eta2]; \%azimuthal angle

vec=[-90:5:90]; \%making 36 bins of 5_interval

(delta n, dx) = hist(delta, vec); \%histogram showing polar angle distribution

(eta n,dx) = hist(eta, vec); \%histagram showing azimuthal angle distributiion

delta $n=$ delta $n^{\prime}$ \%placing theta in column matrix

eta n = eta n' \%placing phi in column matrix

sprintf('Calculation time: \%f seconds ',cputime-t)

\section{METHOD OF ANALYSIS}

Our observations (real observed dataset) are compared with the isotropic distribution curves (obtained from simulation) in both $\theta$ and $\phi$ distributions. For this comparison we use three different statistical tests: chi-square-, Fourier-, and auto correlation-test.

\section{Chi-square test}

The $c^{2}$ test is a measure of how well a theoretical probability distribution fits a set of data. $c^{2}$ test is an objective way to examine whether the observational distribution deviates from the expected distribution (in our case, to the isotropic distribution), which is based on the reduced $c^{2}$ value given by,

$$
\begin{gathered}
\chi_{v}^{2}=\frac{\chi^{2}}{v} \\
\chi^{2}=\sum_{i=1}^{n} \frac{\left(N_{0 i}-N_{\dot{e}}\right)^{2}}{N_{\dot{e}}}
\end{gathered}
$$

Here, $n$ represents the number of bins, $N_{o i}$ and $N_{e i}$ represent the observed and expected isotropic distributions and $v$ is the degree of freedom $(v=$ $n-1)$. For an isotropic distribution, the $\chi_{v}^{2}$ value is expected to be nearly zero. The quantity $\mathrm{P}\left(>\chi_{v}^{2}\right)$ gives the probability that the observed $\chi_{v}^{2}$ value is realised by the isotropic distribution. The observed distribution is more consistent with the expected isotropic distribution when $\mathrm{P}\left(>\chi_{v}^{2}\right)$ is larger. We set $\mathrm{P}\left(>\chi_{v}^{2}\right)=0.050$ as the critical value to discriminate isotropy from anisotropy, it corresponds to the deviation from the isotropy at $2 \sigma$ level.

\section{Fourier test}

If the deviation from isotropy is only slowly varying with $\theta$ or $\phi$, the Fourier test can be applied. A method of expanding a function by expressing it as an infinite series of periodic functions (sines and cosines) is called Fourier series. The frequencies of the sines and cosines are increased by a constant factor with each successive term. The general mathematical form of the Fourier series is:

$f(x)=\frac{a_{0}}{2}+\left(a_{1} \cos x+b_{3} \sin x\right)+\left(a_{2} \dot{\cos } 2 x+b_{2} \sin 2 x\right)+\cdots$

The eonstants $a_{0}, a_{1}, b_{1}, \ldots \ldots .$. etc., called Fourier coefficients, are obtained by the formulae: 


$$
\begin{aligned}
& a_{0}=\frac{1}{\pi} \int_{-\pi}^{\pi} f(x) d \\
& a_{n}=\frac{1}{\pi} \int_{-\pi}^{\pi} f(x) \dot{\cos } n x d x \\
& b_{n}=\frac{1}{\pi} \int_{-\pi}^{\pi} f(x) \dot{\sin } n x d x
\end{aligned}
$$

We compare the observed distributions of the polar and azimuthal angle $(\theta$ and $\phi)$ of the galaxy rotation axis with the expected isotropic distributions. If the deviation from isotropy is a slowly varying function of the angle $\theta$ (or $\phi$ ), one can use the Fourier test. Let $N$ denote the total number of solutions for galaxies in the sample, $N_{k}$ the number of solutions in the $k^{\text {th }}$ bin, $N_{0}$ the mean number of solutions per bin, and $N_{o k}$ the expected number of solutions in the $k^{\text {th }}$ bin. Then the Fourier series (equation 8) becomes

$$
N_{k}=N_{0}\left(1+\Delta_{1} \dot{\cos } 2 \theta_{k}+\Delta_{z} \ddot{\sin } 2 \theta_{k}+\Delta_{\varepsilon} \dot{\cos } 4 \theta_{k}+\Delta_{2} \ddot{\sin } 4 \theta_{k}+\cdots\right)
$$

Here the angle $q_{k}$ represents the polar angle in the $k$ th bin. Taking first order Fourier mode,

$$
N_{k}=N_{0}\left(1+\Delta_{1} \dot{\cos } \ddot{2} \theta_{k}+\Delta_{2} \ddot{\sin } 2 \theta_{k}\right)
$$

Here the Fourier coefficients $D_{11}$ and $D_{21}$ are the parameters of the distributions. We obtain the following expressions for the Fourier coefficients $D_{11}$ and $\mathrm{D}_{21}$

$$
\begin{aligned}
& \Delta_{\mathbb{1}}=\frac{\sum_{k=1}^{n}\left(N_{k}-N_{0 k}\right) \dot{\cos } 2 \theta_{k}}{\sum_{k=1}^{n} N_{0 k} \dot{\cos } \ddot{\mathrm{s}}^{2} 2 \theta_{k}} \\
& \Delta_{z}=\frac{\sum_{k=1}^{n}\left(N_{k}-N_{0 k}\right) \dot{\sin } 2 \theta_{k}}{\sum_{k=1}^{n} N_{0 k} \ddot{\sin }^{2} 2 \theta_{k}}
\end{aligned}
$$

The standard deviations $\sigma\left(\mathrm{D}_{11}\right)$ and $\sigma\left(\mathrm{D}_{21}\right)$ can be obtained using the expressions

$$
\begin{aligned}
& \sigma\left(\Delta_{\sharp}\right)=\left(\sum_{k=1}^{n} N_{0 k} \dot{\cos } \dot{\mathrm{s}}^{2} 2 \theta_{k}\right)^{-1 / 2} \\
& \sigma\left(\Delta_{\mathbb{Z}}\right)=\left(\sum_{k=1}^{n} N_{0 k} \ddot{\sin }^{2} 2 \theta_{k}\right)^{-1 / 2}
\end{aligned}
$$

The probability that the amplitude

$$
\Delta_{1}=\left(\Delta_{1}^{2}+\Delta_{z}^{2}\right)^{1+2}
$$

is greater than a certain chosen value is given by the formula

$$
P\left(>\Delta_{1}\right)=\exp \left(-\frac{n}{4} N_{0} \Delta_{1}^{2}\right),
$$

with standard deviation

$$
\sigma\left(\Delta_{1}\right)=\left(\frac{2}{\mathbb{I N} \mathbb{N}_{0}}\right)^{1 / 2}
$$

The Fourier coefficient $\mathrm{D}_{11}$ is very important because it gives the direction of departure from isotropy. In the analysis of the polar angle $(q)$, if $D_{11}<0$ an excess of galaxies with rotation axis parallel to the reference plane is present, whereas for $\mathrm{D}_{11}>0$ the rotation axis tend to be perpendicular to that plane. In the analysis of the azimuthal angle (f), if $\mathrm{D}_{11}<0$ an excess of galaxies with rotation axis projections perpendicular to the direction of the Virgo cluster center, whereas with $\mathrm{D}_{11}>0$ the projections tend to be parallel to that direction. The first order Fourier probability function $P\left(>D_{1}\right)$ estimates the depth of the preferred orientation. The first order Fourier probablity function $P\left(>\mathrm{D}_{11}\right)$ estimates whether (smaller value of $P\left(>\mathrm{D}_{1}\right)$ ) or not (higher value of $P\left(>\mathrm{D}_{1}\right)$ ) a pronounced preferred orientation occurs in the sample. We set $P\left(>\mathrm{D}_{11}\right)=0.150$ as a critical value.

\section{Auto correlation test}

Auto correlation test is a measure of a degree to which there is a linear relationship between two variables. In our case, it takes account the correlation between the number of galaxies in adjoining angular bins. The correlation function is

$$
C=\sum_{1}^{n} \frac{\left(N_{k}-N_{0 k}\right)\left(N_{k+1}-N_{0 k+1}\right)}{\left(N_{0 k} N_{0 k+1}\right)^{1 / 2}}
$$

with the standard deviation

$$
\sigma(C)=(n)^{1 / 2}
$$

In an isotropic distribution any correlation vanishes, so we expect to have $C \rightarrow 0$ 
Reliability of Statistics in Galaxy Orientation Study

It is clear from the expression (9) that the $c^{2}$ value increases when the number of solutions per bin for both the observed $\left(N_{0 i}\right)$ and the expected $\left(N_{e i}\right)$ distributions is increased by the same factor. As the $c^{2}$ value increases, $\mathrm{P}\left(>c^{2}\right)$ decreases. So, our result 'isotropic' can change into 'anisotropic' if the database is filled with additional galaxies. However, the result 'anisotropic' remains the same even if the database increases. Because of the incompleteness of database the numbers we deal with are lower limits. So, our result 'anisotropic' can be seen as more reliable than the result 'isotropic'. The same will happen in the case of the correlation coefficient $C$ and the Fourier probability function $P\left(>\mathrm{D}_{1}\right)$. However, the Fourier coefficient $\mathrm{D}_{11} / \sigma\left(\mathrm{D}_{11}\right)$ value remains unchanged even if the database increases.

\section{RESULTS \& DISCUSSION}

Any deviation from expected isotropic distribution will be tested using four statistical parameters, namely chi-square probability $\left(\mathrm{P}>\mathrm{c}^{2}\right)$, autocorrelation coefficient $(\mathrm{C} / \mathrm{C}(\sigma))$, first order Fourier coefficient $\left(\mathrm{D}_{11} / \sigma\left(\mathrm{D}_{11}\right)\right)$ and first order Fourier probability $\left(\mathrm{P}>\mathrm{D}_{1}\right)$. For anisotropy, the limit of chi-square probability $\mathrm{P}\left(>\mathrm{c}^{2}\right)$ is $<0.050$, auto correlation coefficient $(\mathrm{C} / \mathrm{C}(\sigma))$ is $>1.0$, first order Fourier coefficient $\left(\mathrm{D}_{11} / \sigma\left(\mathrm{D}_{11}\right)\right)$ is $>1.5$ and Fourier probability $\mathrm{P}\left(>\mathrm{D}_{1}\right)$ is $<0.150$ respectively. Any 'hump' (more solutions than the expected) or 'dip' (less solutions than the expected) in the histogram will be discussed as a local effect in the samples. The statistics for the polar and azimuthal angle distributions is given in Table 1 and Table 2 respectively. In the statistics of $\theta$, a negative value of first order Fourier coefficient suggests that the spin vectors of galaxies tend to be oriented perpendicular with respect to the equatorial coordinate system. Similarly, a positive value of first order Fourier coefficient suggests that the spin vectors of galaxies tend to be oriented parallel with respect to the equatorial coordinate system. Whereas, in the statistics of $\phi$, a positive $\left(\mathrm{D}_{11} / \sigma\left(\mathrm{D}_{11}\right)\right.$ with significant value suggests that the spin vector projections of galaxies tend to point radially with respect to the center of the equatorial coordinate system. Similarly, a significant negative value of $\left(D_{11} / \sigma\left(D_{11}\right)\right.$ implies that the spin vector projection of galaxies tend to orient tangentially with respect to the equatorial coordinate system.

In addition to the statistical tests, we also study the 'humps' (bins with more solutions than the expected) and 'dips' (bins with less solutions than the expected) in the polar and azimuthal angle distributions. The solid curve, in the histogram of the $\theta$-distribution, represents the expected isotropic distribution whereas dashed curve is the cosine distribution. The solid circles with $\pm 1 \sigma$ error bars represent the observed distribution. The shaded portion represents the range $0^{\circ}<\theta<45^{\circ}$. A hump (or dip) in the smaller $\theta$ suggests that the spin vectors of galaxies tend to orient parallel (or perpendicular) with respect to the equatorial coordinate system.

Table 1: Statistics of the polar (below) and azimuthal angle (next, see page 7) distributions of $u$-magnitude SDSS galaxies having redshift in the range 0.10 to 0.11 in the subsamples. The $\mathrm{P}\left(>\mathrm{c}^{2}\right)$ represents the chi-square probability (second column). Similarly, $\mathrm{C} / \mathrm{C}(\sigma)$ represents the auto-correlation coefficient (third column). The last two columns give the first order Fourier coefficient $\left(\mathrm{D}_{11} / \sigma\left(\mathrm{D}_{11}\right)\right.$ and first order Fourier probability $\mathrm{P}\left(>\mathrm{D}_{1}\right)$.

\begin{tabular}{llccc}
\hline sample & $\mathrm{P}\left(>\chi^{2}\right)$ & $\mathrm{C} / \mathrm{C}(\sigma)$ & $\Delta_{11} / \sigma\left(\Delta_{11}\right)$ & $\mathrm{P}\left(>\Delta_{1}\right)$ \\
\hline$u 01$ & 0.217 & +0.4 & -1.0 & 0.568 \\
$u 02$ & 0.592 & -0.9 & +0.7 & 0.775 \\
$u 03$ & 0.300 & -0.7 & +0.0 & 0.990 \\
$u 04$ & 0.968 & -0.2 & +0.2 & 0.931 \\
$u 05$ & 0.193 & -1.8 & +0.2 & 0.975 \\
$u 06$ & 0.512 & -1.0 & +0.0 & 0.977 \\
$u 07$ & 0.312 & -0.5 & +0.6 & 0.741 \\
$u 08$ & 0.368 & -0.9 & -0.6 & 0.818 \\
$u 09$ & 0.358 & +0.0 & -0.1 & 0.982 \\
$u 10$ & 0.544 & -0.8 & -0.7 & 0.727 \\
$u 11$ & 0.601 & -0.7 & +0.0 & 0.976 \\
$u 12$ & 0.453 & +0.4 & -0.5 & 0.859 \\
$u 13$ & 0.818 & +0.7 & +0.3 & 0.936 \\
$u 14$ & 0.032 & +4.4 & -3.1 & 0.006 \\
$u 15$ & 0.793 & +0.5 & -0.8 & 0.503 \\
$u 16$ & 0.886 & -0.5 & -0.5 & 0.831 \\
$u 17$ & 0.254 & -1.7 & -0.4 & 0.846 \\
$u 18$ & 0.449 & -1.5 & +0.8 & 0.734 \\
$u 19$ & 0.305 & +0.6 & +0.3 & 0.927 \\
$u 20$ & 0.481 & -0.4 & +0.4 & 0.908 \\
\hline
\end{tabular}




\begin{tabular}{llccc}
\hline & & & & \\
\hline sample & $\mathrm{P}\left(>\lambda^{2}\right)$ & $\mathrm{C} / \mathrm{C}(\sigma)$ & $\Delta_{11} / \sigma\left(\Delta_{11}\right)$ & $\mathrm{P}\left(>\Delta_{1}\right)$ \\
\hline$u 01$ & 0.040 & -1.2 & +0.2 & 0.659 \\
$u 02$ & 0.489 & +0.2 & -1.2 & 0.250 \\
$u 03$ & 0.449 & -0.5 & +0.3 & 0.278 \\
$u 04$ & 0.586 & -1.7 & -0.9 & 0.602 \\
$u 05$ & 0.623 & -0.8 & +0.3 & 0.438 \\
$u 06$ & 0.599 & +0.1 & +1.0 & 0.571 \\
$u 07$ & 0.138 & -1.3 & +1.1 & 0.364 \\
$u 08$ & 0.023 & +0.2 & +0.5 & 0.119 \\
$u 09$ & 0.339 & +0.4 & +0.4 & 0.458 \\
$u 10$ & 0.729 & +0.3 & +0.2 & 0.265 \\
$u 11$ & 0.481 & +0.6 & +0.5 & 0.358 \\
$u 12$ & 0.501 & +0.3 & +1.1 & 0.487 \\
$u 13$ & 0.943 & +0.1 & +0.1 & 0.472 \\
$u 14$ & 0.736 & -0.6 & -0.3 & 0.936 \\
$u 15$ & 0.504 & -0.4 & +1.6 & 0.233 \\
$u 16$ & 0.982 & +0.2 & -0.4 & 0.897 \\
$u 17$ & 0.753 & +0.0 & -0.9 & 0.608 \\
$u 18$ & 0.212 & -1.2 & +0.0 & 0.993 \\
$u 19$ & 0.923 & +0.0 & -0.6 & 0.665 \\
$u 20$ & 0.873 & -0.6 & +0.3 & 0.951 \\
\hline & & & &
\end{tabular}

Similarly, a hump (or dip) in the larger $\phi$ indicates that the spin vectors of galaxies tend to be oriented perpendicular with respect to the equatorial coordinate system. In the histogram of the $\phi$-distribution, solid curve represents the expected isotropic distribution whereas dashed curve is the average distribution. The solid circles with $\pm 1 \sigma$ error bars represent the observed distribution. The shaded portion represents the range $-45^{\circ}<$ $\phi<+45^{\circ}$. The humps and dips in the histograms of $\phi$-distribution are not so easy to interpret as compared to $\phi$-distributions. It is because the range of $\phi$ is $-90^{\circ}$ to $+90^{\circ}$. In the histogram of the $\phi$-distribution, $\phi=0^{\circ}$ means spin vector projections tend to point radially towards the center of the equatorial coordinate system. A hump in the middle (central eight bins) of the histogram suggests that the spin vector projections of galaxies tend to point towards the center of the chosen co-ordinate system. Similarly, a hump at first four and last four bins indicates that the spin vectors projections of galaxies tend to be oriented tangentially with respect to the chosen reference co-ordinate system.
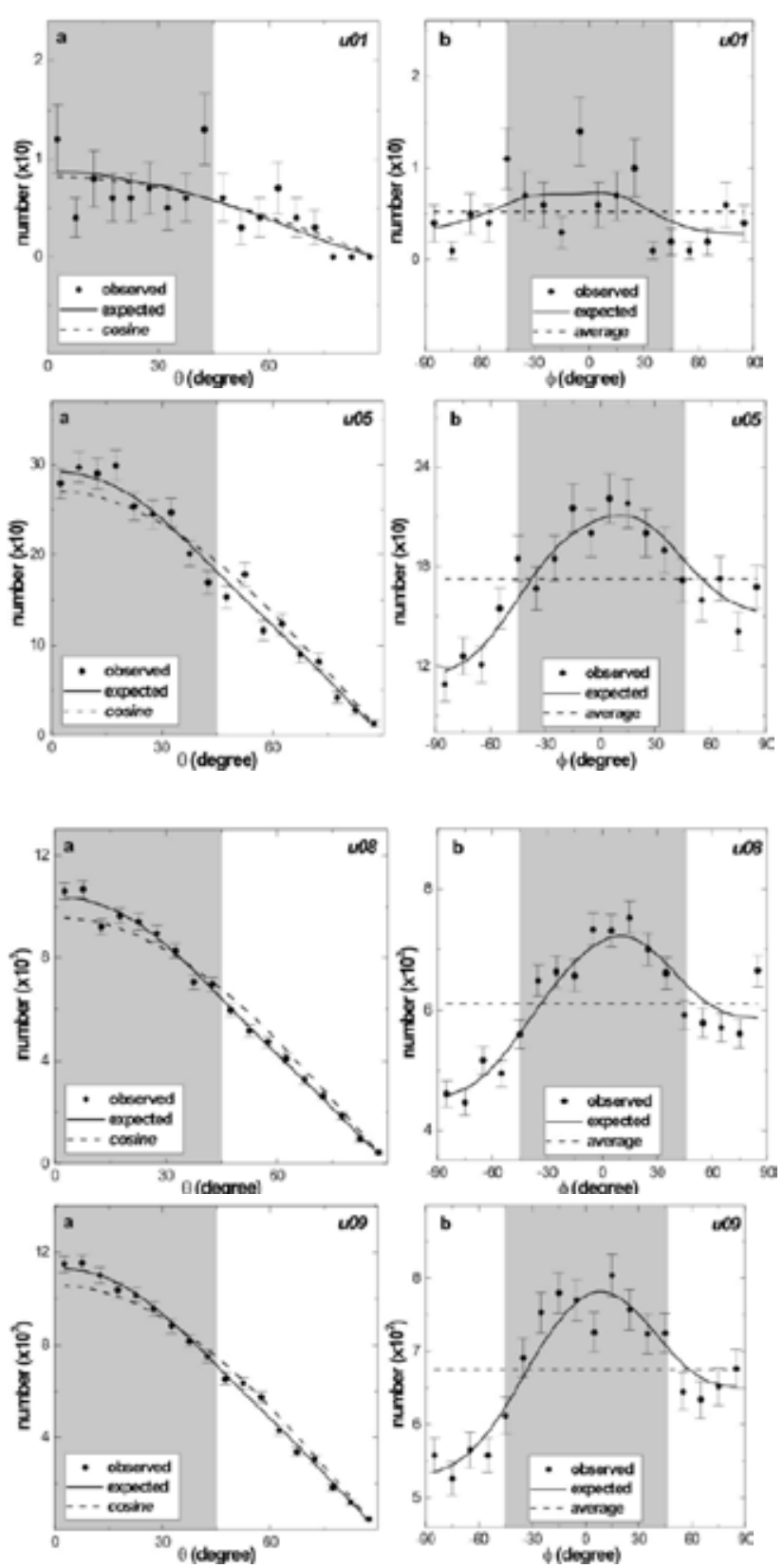

Figure 3: The polar $(\theta)$ and azimuthal angle $(\phi)$ distributions of SDSS galaxies having $u$-magnitude. The solid line represents the expected isotropic distributions. The cosine and average distribution (dashed) is shown for the comparison. The statistical error $( \pm 1 \sigma)$ bars are shown for the observed counts.

Table 1 shows the statistics of the polar angle distribution of galaxies in samples with respect to equatorial coordinate system. The statistics for the polar angle distribution in the sample $u 01$ shows the value of chi-square probability $(\mathrm{P}(>\mathrm{c} 2))$ to be 0.217 i.e. $21.7 \%$. Table 1 shows the statistics of the polar angle distribution of galaxies in samples with respect 
to equatorial coordinate system. The statistics for the polar angle distribution in the sample $u 01$ shows the value of chi-square probability $(\mathrm{P}(>\mathrm{c} 2))$ to be 0.217 i.e. $21.7 \%$ (greater than the significant level 0.050 i.e. $5.0 \%$ ). The auto-correlation coefficient $(\mathrm{C} / \mathrm{C}(\sigma))$ is found to be 0.4 (less than the limit $1 \sigma)$. The first order Fourier coefficient $\left(\mathrm{D}_{11} / \sigma\left(\mathrm{D}_{11}\right)\right)$ is found to be less than the limit $1.5 \sigma$. The first order Fourier probability $\left(\mathrm{P}>\left(\mathrm{D}_{1}\right)\right)$ is found to be 0.568 i.e., $56.8 \%$ (more than $15 \%$ limit). All the statistics suggest isotropy.

The polar angle distribution of galaxies in the sample $u 01$ is shown in the Fig. 3. The number of observed and expected solutions for small angle $\left(<45^{\circ}\right)$ is 54 and 63 respectively i.e. the observed distribution is 9 less than the expected. There is a significant dips at angle $7.5^{\circ}$ with $>2 \sigma$ error limit and no significant humps at angle other than bimodal region. At bimodal region (at $45^{\circ}$ ), the observed solution is greater than the expected solution by 6 because of hump at $42.5^{\circ}$ with $2 \sigma$ error limit. For the large angles (>45_), there is no significant humps and dips. So the number of observed and expected distributions is balanced.

The statistics for the azimuthal angle distribution (Table 1 ) in the sample $u 01$ shows the value of chisquare probability $\left(\mathrm{P}\left(>\mathrm{c}^{2}\right)\right.$ ) to be 0.040 (smaller than the significant level 0.050). The auto-correlation coefficient $(\mathrm{C} / \mathrm{C}(\sigma))$ is found to be -1.2 (smaller than the limit $1 \sigma$ ). The first order Fourier coefficient $\left(\mathrm{D}_{11} / \sigma\left(\mathrm{D}_{11}\right)\right)$ is found to be 0.2 (within the limit $1.5 \sigma)$. The first order Fourier probability $\left(\mathrm{P}>\left(\mathrm{D}_{1}\right)\right)$ is found to be 0.659 i.e. $65.9 \%$ (greater than $15 \%$ limit). Here, chi-square probability $\left(\mathrm{P}\left(>\mathrm{c}^{2}\right)\right)$ and the auto-correlation coefficient $(\mathrm{C} / \mathrm{C}(\sigma))$ shows a weak anisotropy whereas other two statistics show isotropy.

Fig. 3 shows the $\phi$-distribution for the galaxies in the sample $u 01$. A humps are seen at $-45^{\circ},-5^{\circ}(2 \sigma)$ and $75.5^{\circ}$. At angles $-75^{\circ},-10^{\circ}, 35^{\circ}$ and $55^{\circ}$ (with $>2 \sigma$ ) error limit, dips are seen. The numbers of observed and expected solutions are 54 and 55 respectively for central eight bins. These numbers suggest a balance between the observed and expected distributions. In this sample, statistics is rather poor, particularly in the 'dips' of the $\theta$ and $\phi$-distributions where the number of solution is less than seven. Thus we do not conclude isotropy in this sample.

Similar to this, other samples showed isotropy, in general, in the polar and azimuthal angle distributions. The distribution of spin vector and spin vector projections of galaxies are found to be random in all subsamples, except in the subsample u14. In general, our results support Hierarchy model (Peeble 1969). The galaxies that have magnitudes in the range 20.46 to 20.69 (subsample $u 14$ ) showed anisotropy in azimuthal angle distribution. The spin vector orientation of these galaxies tends to oriented parallel to the equitorial plane, suggesting pancake model. It is probably due to strong tidal connection between the galaxies of this group. The cause of this anisotropy should be studied in the future.

Table 2: Statistics of the polar (left) and azimuthal angle (right) distributions of $r$-magnitude SDSS galaxies having redshift in the range 0.10 to 0.11 . The $\mathrm{P}\left(>\mathrm{c}^{2}\right)$ represents the chi-square probability (second column). Similarly, $\mathrm{C} / \mathrm{C}(\sigma)$ represents the auto-correlation coefficient (third column). The last two columns give the first order Fourier coefficient $\left(D_{11} / \sigma\left(D_{11}\right)\right.$ and first order Fourier probability $\mathrm{P}\left(>\mathrm{D}_{1}\right)$.

\begin{tabular}{llccc}
\hline sample & $\mathrm{P}\left(>\lambda^{2}\right)$ & $\mathrm{C} / \mathrm{C}(\sigma)$ & $\Delta_{11} / \sigma\left(\Delta_{11}\right)$ & $\mathrm{P}\left(>\Delta_{1}\right)$ \\
\hline$r 01$ & 0.308 & -0.2 & +0.4 & 0.907 \\
$r 02$ & 0.827 & +0.3 & +1.8 & 0.157 \\
$r 03$ & 0.670 & +0.2 & +0.0 & 0.929 \\
$r 04$ & 0.744 & -0.7 & -0.5 & 0.842 \\
$r 05$ & 0.971 & +0.1 & -0.0 & 0.982 \\
$r 06$ & 0.469 & -1.0 & +0.2 & 0.954 \\
$r 07$ & 0.188 & -0.2 & -0.6 & 0.805 \\
$r 08$ & 0.930 & +0.4 & +0.4 & 0.906 \\
$r 09$ & 0.684 & -1.1 & +0.2 & 0.809 \\
$r 10$ & 0.354 & +0.6 & +0.1 & 0.878 \\
$r 11$ & 0.519 & -0.5 & +0.0 & 0.878 \\
$r 12$ & 0.744 & -0.7 & -0.5 & 0.842 \\
$r 13$ & 0.555 & -0.4 & +0.2 & 0.872 \\
$r 14$ & 0.121 & -0.2 & -1.5 & 0.207 \\
$r 15$ & 0.432 & +1.6 & -0.1 & 0.998 \\
$r 16$ & 0.568 & -1.1 & -0.7 & 0.735 \\
$r 17$ & 0.771 & -0.9 & -0.1 & 0.976 \\
$r 18$ & 0.221 & -1.7 & +0.3 & 0.958 \\
$r 19$ & 0.141 & -1.7 & -0.2 & 0.971 \\
$r 20$ & 0.391 & +1.4 & -0.0 & 0.929 \\
$r 21$ & 0.993 & +0.5 & -0.2 & 0.804 \\
$r 22$ & 0.640 & -0.1 & +0.4 & 0.913 \\
\hline
\end{tabular}




\begin{tabular}{llccc}
\hline sample & $\mathrm{P}\left(>\chi^{2}\right)$ & $\mathrm{C} / \mathrm{C}(\sigma)$ & $\Delta_{11} / \sigma\left(\Delta_{11}\right)$ & $\mathrm{P}\left(>\Delta_{1}\right)$ \\
\hline$r 01$ & 0.142 & +1.9 & -0.9 & 0.182 \\
$r 02$ & 0.109 & +1.7 & -0.7 & 0.160 \\
$r 03$ & 0.892 & -0.2 & -0.2 & 0.741 \\
$r 04$ & 0.108 & +1.0 & +2.2 & 0.036 \\
$r 05$ & 0.911 & -0.5 & -0.0 & 0.818 \\
$r 06$ & 0.836 & -0.8 & -0.6 & 0.622 \\
$r 07$ & 0.927 & +0.1 & +0.9 & 0.491 \\
$r 08$ & 0.073 & +1.4 & +2.5 & 0.026 \\
$r 09$ & 0.954 & -1.0 & +0.1 & 0.933 \\
$r 10$ & 0.327 & +0.4 & +0.6 & 0.283 \\
$r 11$ & 0.197 & +0.0 & +0.0 & 0.255 \\
$r 12$ & 0.108 & +1.0 & +2.2 & 0.036 \\
$r 13$ & 0.140 & -0.2 & +1.1 & 0.551 \\
$r 14$ & 0.450 & +0.5 & -0.2 & 0.248 \\
$r 15$ & 0.263 & +0.7 & +0.7 & 0.473 \\
$r 16$ & 0.684 & +0.2 & +0.6 & 0.535 \\
$r 17$ & 0.592 & -0.5 & -1.3 & 0.342 \\
$r 18$ & 0.353 & -0.3 & -0.3 & 0.415 \\
$r 19$ & 0.001 & +3.3 & +1.7 & 0.019 \\
$r 20$ & 0.128 & +0.3 & +0.9 & 0.621 \\
$r 21$ & 0.761 & +0.7 & +1.1 & 0.534 \\
$r 22$ & 0.608 & -0.7 & -0.9 & 0.645 \\
\hline & & & &
\end{tabular}

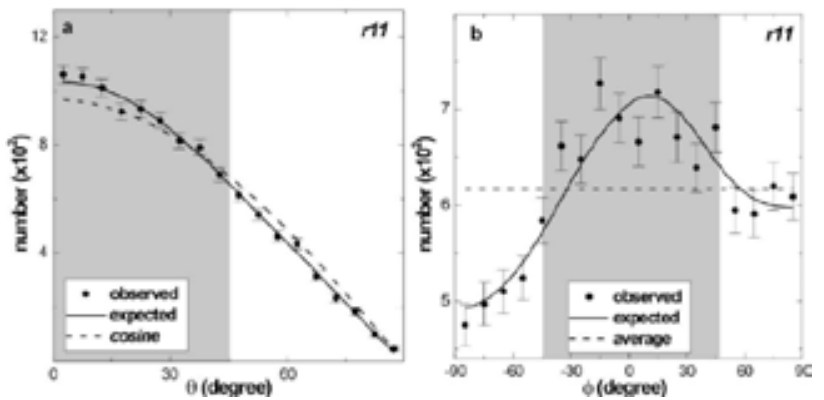

Figure 4: The polar $(\theta)$ and azimuthal angle $(\phi)$ distributions of SDSS galaxies having $u$-magnitude. The solid line represents the expected isotropic distributions. The cosine and average distribution (dashed) is shown for the comparison. The statistical error $( \pm 1 \sigma)$ bars are shown for the observed counts.

No preferred alignment is noticed in the spatial orientation of spin vectors of $\mathrm{r}$-magnitude SDSS galaxies that have redshift in the range 0.10 to 0.11 , suggesting Hierarchy model. However, local effects are found in the samples $r 02, r 04, r 07, r 14$ and $r 19$. In these samples, we suspect a local tidal connection between the rotation axes of galaxies or due to the merging process, the angular momentum vector of few galaxies get affected. A random alignment of spin vector projection of galaxies is noticed in all samples suggesting Hierarchy model and supporting the better reliability of reference co-ordinate system.

\section{CONCLUSION}

We have studied the $r$ and $u$-magnitude dependence in the preferred alignment of spin vector orientation of 44,749 SDSS galaxies that have redshift in the range 0.10 to 0.11 . The $r$ and $u$-magnitude is sensitive towards Lymen, Balmer and Paschan lines of Hydrogen atoms. The emission from these lines is a characteristics of huge HII region or AGN activity in the galaxy. We used the method proposed by Flin \& Godlowski (1986) in order to compute two-dimensional data to three dimensional galaxy rotation axis (polar \& azimuthal angles). We have carried out random simulation by creating $10^{7}$ virtual galaxies and adopting the method proposed by Aryal \& Saurer (2000) in order to find theoretical distribution of galaxy rotation axes. We have compared the differences between theoretical 
distributions and observed distributions using three statistics, namely chi-square, auto-correlation and the Fourier. We conclude our results as follows:

1. The distribution of spin vector and spin vector projections of $u$-magnitude SDSS galaxies that have redshift in the range 0.10 to 0.11 are found to be random in all subsamples, except in the subsample $u 14$. In general, our results support Hierarchy model (Peeble 1969). The galaxies that have magnitudes in the range 20.46 to 20.69 (subsample 414 ) show anisotropy in azimuthal angle distribution. The spin vector orientation of these galaxies tends to oriented parallel to the equatorial plane, suggesting pancake model. It is probably due to strong tidal connection between the galaxies of this group. The cause of this anisotropy should be studied in the future.

2. No preferred alignment is noticed in the spatial orientation of spin vectors of galaxies, suggesting Hierarchy model, as suggested by Peebles (1969). However, a local effect is noticed in the samples $r 02, r 04, r 07, r 14$ and $r 19$. In these samples, we suspect a local tidal connection between the rotation axes of galaxies or due to the merging process, the angular momentum vector of few galaxies get distorted. The luminosity profile of these groups should be studied in order to understand the effect of intra galactic medium.

3. A weak trend is noticed in the spatial distribution of spin vectors of $r$-magnitude galaxies. The low $r$-magnitude (bright) galaxies seem to support Pancake model and high $r$-magnitude (faint) galaxies support Primordial vorticity theory. This trend is not prominent within our redshift limit. Probably, this trend can be tested if we have a database of galaxies that have r-magnitudes from 9 to 35 .

4. A local effect that causes the humps and dips in the angular momentum distribution is observed in different subsamples. In the deep field, density fluctuation is expected and observed in the local scale. Existence of superclusters in the region of interest cannot be ruled out.
5. We have used equatorial system as a physical reference in order to study non-random effects concerning galaxy orientation. Hierarchy model predicts that the choice of co-ordinate system do not alter preferred alignments. However pancake model advocates the importance of physical reference system. We found mixture results: in most cases Hierarchy model and in a few cases Pancake model. This problem should be addressed by using another system such as Galactic or Supergalactic systems.

6. A random simulation for $10^{7}$ virtual particles (i.e, galaxies) carried out successfully by using 4 GB RAM pc. We intend to carry out simulation generating $10^{8}$ virtual galaxies in order to find out sufficiently smooth expected isotropic distribution curves in the future.

In general, our results support Hierarchy model. This model predicts that the spatial orientation of angular vectors of a system of rotationally supported large scale structure (eg. Spiral galaxies) should vanish. In order words, these vectors should align randomly. The spatial orientation of galaxies in 112 clusters were studied till date (Godlowski et al. 1986-2011; Flin et al. 2001, Aryal et al. 2004-2011). In most cases no preferred alignment is noticed (Hierarchy model). However, few clusters strongly support Pancake model. Aryal et al. (2007) noticed a systematic change in the galaxy alignments from early-type (BM I) to late-type (BM III) clusters. This result suggests that the spiral-rich (late-type) clusters (BM II-III and BM III) show a preferred alignment than that of elliptical-rich (early-type) clusters.

\section{ACKNOWLEDGEMENTS}

This research has made use of the SDSS database. We acknowledge Prof. Ronald Weinberger for critical discussions. One author (SNY) acknowledges Central Department of Physics, Tribhuvan University, Kirtipur, for providing various forms of support for his doctoral thesis. We thank Mr. Rajesh Kumar Bachchan for his help during data compilation. 


\section{REFERENCES}

1. Abell, G.O., Corwin, H.G., Olowin, R.P.: Astrophys. J. Supp. 70, 1 (1989)

2. Acharya B., Anisotropy in the spatial orientation of complete search limit SDSS galaxies having redshift $0.10<z<0.11$, M. Sc. (Physics) Dissertation, Tribhuvan University, Kirtipur, Nepal (2012)

3. Aryal B., Bhattarai H., Dhakal S., Rajbahak C. \& Saurer W.: Monthly Notice of Royal Astron. Soc. 434, 1939 (2013)

4. Aryal B., Yadav S. N. \& Saurer W., Bulletin of Astron. Astron. Soc. Ind. (BASI), 40, 65 (2012)

5. Aryal, B., Paudel, S., Saurer, W.: Astronom. Astrophys. 479, 397 (2008)

6. Aryal, B., Saurer, W.: Monthly Notices Royal Astron. Soc. 366, 438 (2006)

7. Aryal, B., Saurer, W.: Astronom. Astrophys. 432, $841(2005 a)$

8. Aryal, B., Saurer, W.: Astronom. Astrophys. 432, 431 (2005b)

9. Aryal, B., Saurer, W.: Astronom. Astrophys. 425, 871 (2004)

10. Aryal, B., Saurer, W.: Astronom. Astrophys. lett. 364, L97 (2000)

11. Colless M., et. al., Monthly Notices Royal Astron. Soc. 328, 1039 (2001)

12. Doroshkevich A. G., Shandarin S. F.: Monthly Notices Royal Astron. Soc. 184, 643 (1978)
13. Doroshkevich A. G., Astrophysics, 6, 320 (1970)

14. Doroshkevich A. G.: Astrophysical Journ. 14, L11 (1973)

15. Flin P., Godlowski W.: Monthly Notices Royal Astron. Soc. 222, 525 (1986)

16. Flin P.: Monthly Notices Royal Astron. Soc. 325, 49 (2001)

17. Gamow G., Phys. Rev. 86, 251 (1952)

18. Godlowski, W.: Monthly Notices Royal Astron. Soc. 265, 874 (1993)

19. Godlowski, W.: Monthly Notices Royal Astron. Soc. 271, 19 (1994)

20. Heidmann J., Heidmann N., de Vaucouleurs G.: Monthly Notices Royal Astron. Soc. 75, 85 (1971)

21. Ozernoy L. M., in Longair M. S., Einasto J., eds, Proc. IAU Symp. 79, The Large Scale Structure of the Universe. Reidel, Dordrecht (1978)

22. Padmanabhan T., An Invitation to Astrophysics, World Scientific Publishing Co. Pvt.Ltd. (2006)

23. Peebles P. J. E.: Astrophysical Journ. 142, 1317 (1965)

24. Peebles P. J. E.: Astrophysical Journ. 155, 393 (1969)

25. Stein R.: Astron. \& Astrophys. 35, 17 (1974)

26. York, D.G., Adelman, J., Anderson, J.E.: The Sloan Digital Sky Survey: Technical Summary (2000) 